\title{
Double stimulation in healthcare emergencies: fostering expansive, collective tool use through simulation-based continuing professional education
}

\section{Nick Hopwood}

School of International Studies \& Education, University of Technology Sydney, Sydney, Australia

Department of Curriculum Studies, University of Stellenbosch, Stellenbosch, South Africa

Johanna Dahlberg

Department of Health, Medicine and Caring Sciences, Linköping University, Linköping, Sweden

Marie Blomberg

Department of Biomedical and Clinical Sciences, Linköping University, Linköping, Sweden

Department of Obstetrics and Gynecology, Linköping University, Linköping, Sweden

Madeleine Abrandt Dahlgren

Department of Health, Medicine and Caring Sciences, Linköping University, Linköping, Sweden

Correspondence details:

Nick Hopwood

nick.hopwood@uts.edu.au

PO Box 123, Broadway, NSW 2007, Australia

ORCID 0000-0003-2149-5834 


\section{Funding source}

NA

Disclosure statement: N/A-No financial or other interest applies. 


\section{Double stimulation in healthcare emergencies: fostering expansive, collective tool use through simulation-based continuing professional education}

This paper explains how simulation-based continuing professional education can enable professionals to overcome significant challenges in healthcare practice. It focuses on pedagogies that address conflicts of motives experienced by teams at work by promoting collective use of protocols and an auxiliary motive to collaborate in agile, relational practices. Data relating to a simulation program (PROBE) associated with reduced injuries in emergency birth situations are examined. The concept of double stimulation informs analysis of simulated scenarios and linked debriefs. PROBE transforms a commonly used protocol from a memory tool used by individuals to an 'in-between' tool used expansively and collectively across the birthing team. Crucial to this are diverse epistemic levels of mediation that enable teams to resolve conflicted, high-stakes situations through fluid, responsive interactions. Indications in the data that PROBE pedagogies foster transformative agency among health professionals are highlighted and discussed. The paper thus adds to understanding of how double stimulation as a principle of volitional action can be put to work in continuing professional education.

Keywords: emergency care; professional learning; simulation; debriefing; workplace learning; midwifery; birth

\section{Introduction}

How can professional education promote the agile, collective practices that such situations demand? This paper explores how one successful program has accomplished this, highlighting how it addresses a conflict of motives, and transforms the use of a protocol from an individual memory tool to a tool used in collective practice. In doing so, it explores transformative agency by double stimulation (TADS, Sannino this issue) in the context of ongoing professional education in healthcare. 
Protocols are commonly used in healthcare practices to help individuals remember what to do. They are particularly important in emergency situations, and as such a frequent focus on ongoing education and training for professionals. But emergency care situations frequently require practitioners to work together, often in teams constituting diverse health disciplines. The clinical focus in the simulation pedagogies analysed is an emergency situation when a baby gets stuck in the birth canal during birth—referred to as shoulder dystocia.

When a shoulder dystocia occurs, both the mother and child are at risk. "Few obstetric emergencies cause as much anxiety as shoulder dystocia" (Fahey \& Mighty 2008, 121). Shoulder dystocia occurs between two and seventy times per thousand births, depending on foetal and maternal weight (Gobbo et al. 2017). It is unpreventable, unpredictable and often occurs with no warning (Gobbo \& Baxley 2000; Baxley \& Gobbo 2004; Hope et al. 2005; McArdle et al. 2018). It can result in serious maternal or neonatal injury, permanent paralysis, asphyxia or even death (McArdle et al. 2018). Injuries frequently lead to litigation, malpractice allegations and significant compensation payouts by health services (Fahey \& Mighty 2008; Jenkins 2014; McArdle et al. 2018).

For these reasons, continuing professional education focused on shoulder dystocia is crucial—ensuring professionals can respond effectively to this high-stakes situation. This paper examines simulation-based continuing professional education in a site that has accomplished success in reducing adverse outcomes from shoulder dystocia (Dahlberg et al. 2018). Such success that has proved elusive in many contexts.

Shoulder dystocia requires "rapid and well-coordinated intervention by the health care team, some of whom may not have worked together before" (McArdle et al. $2018,192)$. It presents practitioners with a deep conflict of motives: to deliver the baby 
quickly, and to avoid injury to mother and child. Framing the problem on these terms through Vygotskian concepts of double stimulation leads to new insights into protocols that are widely mandated, and thought to function by standardising practice through aiding individual practitioner memory and associated action.

People's understandings of a problem and responses to it change through the use of mediating tools (Vygotsky 1997). The concept of double stimulation holds that an auxiliary stimulus enables us to 'move' when paralysed by a conflict between two equally (un)appealing courses of action. Sannino’s (2015b; Sannino \& Laitinen 2015) model conceptualises how such conflicted situations are resolved through mediation by cultural tools, articulating this as a principle of volitional action underpinning the emergence of transformative agency. This lies at the heart of the idea of transformative agency by double stimulation (TADS).

Transformative agency involves responses to demanding situations through envisioning new possibilities in collective activity, breaking away from established ways of working to transform a situation (Engeström et al. 2014; Virkkunen 2006). This model has been shown to capture important features of the way conflicts of motives encountered in everyday life and work settings are resolved (e.g. Hopwood \& Gottschalk 2017; see also Sannino 2015a). It has not been explored in the context of continuing professional education.

Much of the early literature on double stimulation (including Vygotsky's own writing) focuses on how individuals resolve conflicted situations through culturally available means. More recent research (Sannino 2016) suggests that collective dynamics have an important role to play when groups of people are presented with a common conflicted situation, as is the case for healthcare practitioners responding to shoulder dystocia. 


\section{Shoulder dystocia and double stimulation}

Conceptualising shoulder dystocia in terms of double stimulation recasts how we might understand and deliver continuing professional education aiming to help clinicians respond effectively and reduce adverse outcomes for mothers and new-born babies.

The dilemma of shoulder dystocia can be expressed directly in terms of Sannino's (2015b, this issue) TADS model. There is a conflict of stimuli. One set of stimuli relate to the baby being pushed down the birth canal: contractions, the mother pushing, and (sometimes) a hormonal infusion used to aid vaginal birth by reinforcing contractions. An opposing stimulus occurs when the shoulder lodges against the mother's bone: the baby's head retracts slightly_remaining outside the mother's body but pulling back the other way. The moment the shoulder dystocia is noticed, several actions to deliver the baby must be stopped in order to avoid injury. The mother is asked to stop pushing, any active infusion is switched off, and equipment that has been used to pull the baby out is set aside. There is a five- to seven-minute window to deliver the baby, beyond which there is a significant risk of critical complications, brain injury to the child, or death (Baxley \& Gobbo 2004; Gobbo et al. 2017). This creates an urgent imperative to act at the very moment that normal delivery actions become impossible. "A relatively brief delay in delivery of the shoulders may be associated with a fatal outcome" (Hope et al. 2005, 1256).

There is thus a conflict of motives: the need to deliver the baby, versus the need to avoid injury to the baby and mother. A resolution must be found quickly. Shoulder dystocia "requires a very quick and coordinated response with no time to debate the general merits of one management strategy over another" (Fahey \& Mighty 2008, 121).

While there is some variation in recommended solutions (Chauhan et al. 2010), the HELPERR protocol is in widespread use as a mnemonic and has been incorporated into pre-service and ongoing professional education in many countries. Originally 
developed as part of the Advanced Life Support in Obstetrics (ALSO) course in the USA, it is a: "clinical tool that can provide maternity care providers with a structured framework in which to deal with an extremely difficult and charged situation" (Gobbo \& Baxley 2000, 5). Others have elaborated on this function:

This mnemonic had a number of advantages; in particular it encouraged clinicians to move away from ineffective and potentially dangerous practices such as applying fundal pressure, and introduced an escalating set of manoeuvres designed to increase the space in the pelvis, and to attempt to rotate the baby into an oblique position to resolve the dystocia. The main advantage of the ALSO approach was the use of the mnemonic as an aide memoir for the provision of systematic care. (Jenkins 2014, 319)

HELPERR has become synonymous with shoulder dystocia management in many settings (Jenkins 2014). It is referred to in every regional framework for complex births in Sweden, where the study was conducted. The meaning of each letter is presented in Table 1 (Huntley \& Dickson Smith 2017). Guidelines commonly assert the need to document actions performed and their precise timing (Chauhan et al. 2010). Such tools are often discussed in terms of their capacity to help individuals know what to do, and to produce standard responses:

Management algorithms, such as the RCOG [Royal College of Obstetricians and Gynaecologists] shoulder dystocia algorithm, and documentation proforma... not only help to standardise practice, but also guide staff to undertake the correct actions; making the right way, the easy way. (Cornthwaite et al. 2015, 4). 
Table $1 \quad$ Summary of HELPERR ${ }^{1}$

\begin{tabular}{ll}
\hline Letter & Detail \\
\hline $\mathrm{H}$ & Call for Help (e.g. senior midwife, obstetrician, anaesthetist, paediatrician, \\
& scribe) \\
\hline $\mathrm{E}$ & Evaluate for episiotomy (consider a cut to provide additional room when carrying \\
& out internal manoeuvres) \\
\hline L & Legs into McRoberts' position (flex mother's legs against abdomen, knees \\
& towards ears) \\
\hline P & Pressure (apply external suprapubic pressure known as Rubin I; avoid fundal \\
& pressure) \\
\hline E & Enter (internal rotary manoeuvres performed by inserting fingers past baby's \\
& head; three named manoeuvres can be tried repeatedly with recommendation to \\
& change after 30 seconds: Rubin II, Woods Screw, Reverse Woods Screw) \\
\hline R & Remove posterior arm (flex baby's elbow, sweep forearm across chest and \\
& deliver arm) \\
& \\
& Roll onto all fours (mother onto hands and knees and repeat manoeuvres: Gaskin \\
& \\
&
\end{tabular}

(Gobbo et al. 2017)

In terms of double stimulation, HELPERR ostensibly acts as an auxiliary stimulus, a tool that helps practitioners escape the conflict of motives. Attention is directed to a sequence that offers a means of safe and rapid delivery through actions guided by a new motive to change the interior configuration between the baby and mother. The mnemonic aspect is designed to function as an internalised memory aid that prompts externalisation through individual actions. However, the analysis that 
follows reveals this to be only part of the picture in terms of how double stimulation works in this setting. A fuller understanding reveals insights regarding the value of double stimulation in promoting agile, collective practice in continuing professional educations more widely. The role of education here is not to simply address gaps in professionals' knowledge, or provide opportunities to rehearse and practise skills, but rather to foster transformative agency among professionals.

\section{Research on professional education through simulation of shoulder dystocia}

Merely knowing HELPERR and memorising its meaning is not enough. An evidence review documented problems using HELPERR, including failing to explicitly name shoulder dystocia, inability to complete manoeuvres associated with the second 'E', and confusion over the manoeuvres, which cannot be seen either by the person performing them or the rest of the team (Jenkins 2014). The Confidential Enquiry into Stillbirths and Deaths in Infancy (Hope et al. 2005) and SaFE study (Crofts et al. 2006) found frequent failure to perform and document standard manoeuvres (see also Draycott et al. 2008). Shoulder dystocia is common enough that practitioners are likely to encounter it, but not common enough that practitioners become comfortable in its management without specific, additional training (Fahey \& Mighty 2008). Hence regular continuing professional education for shoulder dystocia has been recommended for over 20 years (Cornthwaite et al. 2015).

Research frequently points to potential benefits of simulation training. A study of 450 shoulder dystocia simulations at Southmead Hospital (UK) found that prior to training there was widespread use of potentially harmful actions (such as fundal pressure), failure to call for relevant help, inadequate communication between team members, and failure to deliver the baby in simulations (Crofts et al. 2008). There were 
significant and lasting improvements in simulated deliveries after training. Draycott et al.'s (2008) analysis of actual births after training in the same hospital found improved clinical management and more frequent use of actions prescribed by HELPERR.

Linked analyses found reduced neonatal injury (see also Crofts et al. 2006) and improvements in knowledge relating to obstetric emergency management (Crofts et al. 2007). Non-participants in an Americanised adaptation of the same program accounted disproportionately for poor outcomes (Weiner et al. 2014). A different simulation-based education program improved physicians' performance of four technical manoeuvres, and communication scores as observed in subsequent simulated shoulder dystocias (Goffman et al. 2008).

Professional education focused on this kind of training gives practitioners experience at managing their response and reduces anxiety, and can lead to measurable reductions in sequelae (especially injury) for both mother and child (Fahey \& Mighty 2008; Crofts et al. 2011). However, this varies, and in one UK hospital injury rates went up after training was introduced, leading the authors to conclude: "not all training is equal in effect" $(2011,12)$. This was echoed by Cornthwaite et al. (2015) and Dahlberg et al. (2018) who noted inconsistent evidence regarding the effect of simulation on clinical outcomes.

One issue that arises repeatedly in relation to varying outcomes concerns the collective nature of managing shoulder dystocia. Cornthwaite et al. argue:

Although training for shoulder dystocia typically focused on the individual skills of the accoucher [midwife], effective multi-professional team working remains essential. Training individuals, rather than teams, may lead to the omission of critical steps. $(2015,3)$ 
There has therefore been a shift away from a focus on individual technique to better team co-ordination and training (Siassakos et al. 2009; Monod et al. 2014). McArdle et al. (2018) found that a simulation program focusing on teamwork and communication skills improved use of shoulder dystocia safety strategies. They noted:

The use of independent checks with cognitive aids such as checklists, algorithms, or protocols can encourage a shared mental model and guide a systematic approach to management of shoulder dystocia and documentation during what is often a chaotic event. $(2018,197)$

This suggests that mnemonic tools can potentially support relational aspects of dealing with emergencies. However, this function remains untheorised, and the pedagogies that would effectively support this are inadequately understood and poorly articulated. This paper addresses this gap by examining practices in a setting where multi-professional simulation education with a specific focus on teamwork (PROBE) has led to significant improvements in clinical outcomes over a decade (Dahlberg et al. 2018).

\section{The PROBE program}

While practitioner knowledge and confidence, and the application of preferred management techniques are important indicators of successful training for shoulder dystocia, patient outcomes are regarded as the 'gold standard' measure (Siassakos et al. 2009). Outcomes of a ten-year simulation ongoing professional education program (PROBE) implemented at Linköping University Hospital in Sweden span all these domains, as summarised in Table 2, which is based on data collected before PROBE (2004-2007) up until 2015. 
Table 2

Summary of outcomes related to PROBE

\begin{tabular}{ll}
\hline Outcome domain & Details \\
\hline Practitioner & Staff confidence in handling shoulder dystocia increased from $48 \%$ to \\
& $62 \%$ \\
\hline Indicators of & Appropriate documentation of delivery (63\% to 93\%); stopping \\
sanctioned practice & hormonal infusion ( $0 \%$ to $54 \%)$ \\
and HELPERR & Use of techniques in HELPERR: more than four-fold increase in \\
manoeuvres & internal rotation of anterior shoulder \\
& Brachial plexus injury in shoulder dystocia births from $73 \%$ to $17 \%$ \\
\hline Patient outcomes & Reductions in fractures to foetus' clavicle and humerus \\
\hline
\end{tabular}

(Dahlberg et al. 2018)

PROBE (Practical Obstetric Team Training [translated from Swedish]) has been running since 2008. The program targets interprofessional teamwork and obstetric emergency skills as connected drivers of improved clinical outcomes (Dahlberg et al. 2018; Hopwood et al. in press). All staff involved in deliveries are required to complete PROBE every 18 months. This involves an afternoon in which teams complete three stations: two simulations and a skills training session. Teams comprise a primary midwife and nursing assistant (who work together in all births), supplemented by a second midwife (who is typically appointed as coordinator of the team) and obstetrician when help is called for $^{2}$. One of the simulations always involves a shoulder dystocia, although the details of the scenario change each year. The skills session covers HELPERR among other protocols.

The simulation uses a mannequin of the mother's pelvis and thighs, and a babylike doll. One facilitator observes the team, making notes to inform the subsequent 
debrief; she also provides information such as CTG readings which have to be 'made up' as part of the simulation. A second facilitator/operator plays the role of the mother, while holding and positioning the baby from behind the mannequin. The scenario runs for around 20 minutes, followed by a 20-minute debriefing that follows a three-round structure. This echoes Steinwachs' (1992) widely used model of description, analysis and application, but breaks away from the detail of this in several important ways, summarised in Table 3. Debriefing practices are highly consistent across the facilitators and have been stable over time.

Table 3 The rounds of debriefing used in PROBE

\begin{tabular}{|c|c|c|}
\hline Round & Steinwachs (1992) model & PROBE approach \\
\hline Description & $\begin{array}{l}\text { Participants describe what happened } \\
\text { to them-experiences and } \\
\text { impressions; listening to others to get } \\
\text { the whole picture }\end{array}$ & $\begin{array}{l}\text { Sequential recapitulation that } \\
\text { emphasises connections in action- } \\
\text { what happened next, what help was } \\
\text { needed, who made decisions etc. }\end{array}$ \\
\hline Analysis & $\begin{array}{l}\text { Also referred to as analogy; } \\
\text { systematic examination of simulation } \\
\text { to identify parallels with the real } \\
\text { world }\end{array}$ & $\begin{array}{l}\text { Focus on identifying what each } \\
\text { member did well and how this } \\
\text { contributed to the team work }\end{array}$ \\
\hline Application & $\begin{array}{l}\text { Participants consider what } \\
\text { understandings are most relevant to } \\
\text { them and what course of action they } \\
\text { wish to carry out as a result }\end{array}$ & $\begin{array}{l}\text { Focus on what participants will take } \\
\text { to the real delivery suite, with } \\
\text { emphasis on how it links to safety } \\
\text { and teamwork }\end{array}$ \\
\hline
\end{tabular}


The outcomes listed in Table 2 show sustained improvements in shoulder dystocia deliveries. PROBE appears to be securing the use of HELPERR as a tool to resolve the conflict of motives and enhance outcomes. What analyses to date have not revealed is how this is accomplished. It is not known how the simulation and debrief work with HELPERR, nor have data relating to PROBE been used as a basis to better conceptualise how double stimulation works with collectives at work.

\section{Data collection and analysis}

In 2018, three shoulder dystocia simulation scenarios and subsequent debriefs were video recorded. Three teams of three or four practitioners completed the shoulder dystocia session. Spoken interactions were transcribed verbatim and then translated into English, with additional notes made about what the participants did physically during the scenario and the material artefacts they used. The facilitators were the same in each session.

Analysis was conducted in two stages, first reviewing each scenario, focusing on how the actors oriented to one another, the mother, and the baby, noting evidence of HELPERR being mentioned or enacted. Second, the three rounds within each debrief (see Table 3) were analysed separately, focusing on the changing way HELPERR mediated practices that enacted a solution to the conflict of motives. Findings will now be presented and discussed to reflect this analytical progression. 


\section{Findings}

\section{The simulation: HELPERR in action}

In each scenario the baby was delivered between 60 and 90 seconds after the slight retraction of the baby's head, the sign that a shoulder dystocia had occurred (Gobbo et al. 2017; Huntley \& Dickson Smith 2017). Table 4 presents transcriptions of this period for each team, pointing to HELPERR as it was enacted. Since the scenario involved a slow labour, help had previously been called for meaning a second midwife and an obstetrician were already present; in some cases, a cut (episiotomy) had already been performed, meaning the emergency management started at ' $L$ ' (see Table 1).

Table 4 How each team managed the shoulder dystocia [see end]

[Insert Table 4 around here]

As soon as the shoulder dystocia was noticed the teams took actions to stop the normal birthing process. The mother was told to stop pushing, and the hormone drip was switched off. In Team 2, HELPERR was named, and in Team 3 the letters from the mnemonic were spoken, while in Team 1 the sequence was followed but no explicit reference was made to HELPERR. The solution to the problem of needing to act quickly without injuring the mother or child was enacted through the prescribed sequence of actions.

Table 4 reveals how HELPERR depends on responsive collective actions if it is to function as an auxiliary device. One person cannot perform the internal manoeuvres $[\mathrm{E}]$ and apply pressure $[\mathrm{P}]$ at the same time, and these two actions need to be attuned to one another. The difficult, tiring internal manoeuvres were performed alternately by a 
midwife and obstetrician in Teams 1 and 3. The invisibility of this work presented a challenge to the other team members. This was overcome by the person performing the second E (internal manoeuvres, see Table 1), verbalising what was happening.

In Vygotskian terms, HELPERR is designed as a tool of remembering that is internalised by an individual. In the simulation this is externalised through bodily actions and commentaries on those actions. By guiding the professionals in terms of the sequence of actions to perform, HELPERR mediated their activity in a particular epistemic way: it was (at this moment) an artefact that enabled participants to address questions of 'How?' and 'In which order?' (Engeström 2007). Scripts, rules, plans and algorithms are characteristic of this epistemic level, and here we see HELPERR functioning as intended, securing a prescribed sequence of actions.

\section{Debrief round 1: Description}

The first round of debrief focused on description (see Table 3). The facilitator began with the first midwife, bringing in other participants according to when they joined the action. The facilitator invited an account of what happened in what order. Where things were missed, the facilitator would rewind, saying "Before you got there, what did you say?", or "You did something before that".

This process produced new artefacts in the form of narratives of what had happened. These mediated collective reflection at a different epistemic level, in terms of 'Who? What? When?' (Engeström 2007). This highlighted connectivity in action in a way that went far beyond multiple individual accounts. Facilitator questions linked one person's account to another: "There was a change in the baby's heart rate and then you [midwife 1] called for the obstetrician. What happened then? [to the Assistant]". Participant accounts highlighted implications of the speaker's actions for others: "Then 
I asked you [midwife1] if the bladder was emptied, and I palpated the patient a bit”. They also articulated what happened in terms of what others did: "Then we closed the drip. You [midwife 1] told us to, and you [midwife 2] did it, I did not have time", "You came to the front shoulder and rear shoulder and it did not work", "They tried to comfort you [the mother]". At other times, participants expressed a collective 'we' as the subject: "What did we say, we said 'Let's run HELPERR"”, “Then we stopped the infusion and up with the legs, and we knew about the bladder". Some comments combined several of these means of linking 'I' to 'you' to 'we': "Then I asked you what you wanted help with, and we agreed on that external pressure was needed... and then we tried it".

By revisiting who, what and when from a collective perspective, the descriptive round connected the epistemic level 'How? In what order?' to that of 'Who, What, When?'. Through this, the participants' understanding expanded from focusing on a sequence of actions to an account that foregrounded a web of connections. This relational understanding provided a foundation for further expansions. Participants started to co-author their accounts of, and reflections on practice, giving a differentcollective - shape to narratives of what had happened. This is an expression of transformative agency (Engeström et al. 2014; Haapasaari et al. 2016).

\section{Debrief round 2: Analysis}

The second debrief round proceeded from description to analysis (Table 3), focusing specifically on what went well: "Now we have agreed that we have about the same picture of what happened. What did I do really well? Or us together?". The facilitator's collective framing 'us together' was repeated across the three team debriefs. She made frequent use of the plural version of 'you' in Swedish, echoed in the collective analysis 
offered by participants: "What we did well, all of us, we communicated well with each other". When participants highlighted individual actions, the facilitator followed up by linking these to their impact on others. This took the co-authoring of practice further, expanding it to include analytical as well as descriptive qualities:

Obstetrician: To be calm, take it easy. I think I did well because you do get stressed, but realistically you do have some time to understand what you have to deal with.

Facilitator: Which contributed to calmness in the room, which gives you [plural] the chance to stop and think where you are [referring to HELPERR].

The collective account of how the emergency was handled was used to expand understandings of why particular actions were important in this accomplishment. This brought another epistemic level (Why?) into play (Engeström 2007). The HELPERR guidance is based on physiology and actions that can be taken to alter and work with the embodied conditions of shoulder dystocia, such as increasing the functional pelvic width (Gobbo \& Baxley 2000; Huntley \& Dickson Smith 2017). However, the second round of debrief focused on how HELPERR emerged not just as a tool used by individual participants, but one used in-between them as they worked as a team (Kuiper 2018). The excerpt below illustrates this in relation to verbalising HELPERR actions.

Midwife 2: What we did well, all of us, we communicated with each other.

Facilitator: Any examples?

Midwife 2: I was thinking, when you were about to do the HELPERR manoeuvre, I was standing trying to repeat out loud what we had already done, so that we would know where we were. It 
is good to communicate so that you know what to do. And you [obstetrician spoke out, 'Now I am doing the cut' and then you [1st midwife] said 'I am trying the front shoulder and the back shoulder'. It's not easy to see.

Team 3's explicit discussion of which letter they were up to (Table 4) shows the importance of this to practitioners in the moment. Verbalisation not only creates a shared understanding of where the team is, but also externalises what cannot be seen as practitioners perform internal manoeuvres. This enables others to apply pressure and move from one side of the mother's body to another as necessary. It is also crucial when one person takes over from another (as happened in Teams 1 and 3). In this way, the practice takes collectively authored, shape and direction. Significantly, the verbalisations that were highlighted and valued in the debrief were not simply those that stated which letter in the sequence had been reached. Rather the focus was on those that made the body 'see-through' (see Hopwood et al. 2014) by giving details of actions that were otherwise invisible.

Practitioners' understandings of why particular actions matter in relation to HELPERR expanded from standardised and physiological to incorporate responsive collective practice mediated by collective tool use. These understandings of 'Why?' themselves contributed to an expanding understanding of how to respond, collectively, to shoulder dystocia. This expansion was driven by introducing new epistemic levels, and connecting them to others, such that the team's activity was mediated across multiple, inter-related epistemic levels. 


\section{Debrief round 3: Application}

The third round in each debrief focused on application. As shown in Table 3, this broke away from the Steinwachs (1992) approach by going beyond individual lessons learned to emphasise visions of and commitments to future action with others. Envisioning and commitment to action are frequently noted as kinds or expressions of transformative agency (Haapasaari \& Kerusuo 2015; Haapasaari et al. 2016; Engeström et al. 2014). The importance of individual actions was repositioned in future-oriented narratives as part of agile, collective practice. HELPERR was imagined in future as a tool used by and in-between team members.

Facilitator: It could be something you felt you did so bloody well you will remember it.

Midwife1: It's teamwork and about communicating clearly with each other, I feel safe about what's happening in the room, that you can ask questions, no, now we are doing this! and I have my role, a coordinator is a little more experienced, everyone has their role and you help each other out, it's safe

The positioning of bodies around the mother was again linked to the importance of verbalising actions: "Then when it is happening, you should say 'we change positions"'. Narrating the future in terms of collective co-authoring helped individuals re-cast their role. Often this related to producing and maintaining calm across the team as in the following sequence, when the Facilitator reinforced how communication between team members can help the practice emerge in helpful ways:

Midwife 2: I take with me to calm down a little, when the obstetrician arrives, to give the time to get an opinion. She already knew we had tried pushing a couple of times, the CTG was on 70 [it should be around 135], now the baby has to come out, that 
was the only thing for me. We have to give time to the obstetrician to make that decision, but for me it was already 'it has to be now', and it's difficult for me to wait.

Facilitator: So was that a problem? The obstetrician said, 'Give me some time', and you did. So I think you communicated about it... It's not only your responsibility [to give time], it's also hers, which she did when she said 'Give me time'.

On the basis of their shared simulation experience and subsequent facilitated discussion, participants also reached generalised principles to apply in future real cases.

Assistant: $\quad$ You should watch the clock. I have not experienced that and didn't realise the importance before.

Acting Mother: Yes, you can always do that, it can happen in an ordinary case as well, if the head is out and the contraction is ending ... I always throw a glance at the watch... and you can say if it's only one minute, I can wait for the next contraction.

In the case of clock-watching, commitments to future action were discussed as something to establish as routine in all deliveries, with the intention that it would be more secure and habitual when emergencies arise and the time-keeping becomes a crucial part of collective practices where the stakes are urgent and high.

The questions asked and answers given produced narratives that shifted the epistemic level of mediation to 'Where to?'. This flowed from prior work, maintaining rich connections to questions of 'How?', 'Who, What, When?', and 'Why?'. This secured the mediating potential of HELPERR through new relational possibilities. Rather than individuals confirming how they could enact a standardised protocol in future, they elaborated how they might work with others in a situationally responsive way. Their visions of the future had significantly expanded, accompanied by concrete 
commitments to future actions. Importantly, although such commitments were grounded in particular times and places ('next time there is a shoulder dystocia and I am on the floor') they were not rigid. This was accomplished by understanding and enacting HELPERR as an auxiliary stimulus that requires fluid relational dynamics in emergent practices. This involved use of this tool being changed from an individual basis to a collective or in-between one (Kuiper 2018).

\section{Discussion}

PROBE harnesses the power of double stimulation as a principle of volitional action, and creates conditions in which transformative agency emerges. It can therefore be understood as a site where pedagogy is fostering TADS. The mixed outcomes of simulation programs elsewhere point to the need for an approach that engages with the collective nature of handling shoulder dystocia. PROBE addresses this by adapting Steinwachs' (1992) three-stage approach to debriefing into a more relationally oriented form.

In the debriefing, HELPERR became a question of co-authoring answers to a specifically relational question: 'How do we work together?'. The solution to the conflict of motives involved co-authored actions that produced agile, connected practice and expanded HELPERR into a tool used between the team members, not just by them: naming the dystocia, articulating the HELPERR steps, verbalising actions, shaping practice through in-the-moment negotiations (e.g. switching who performs the internal manoeuvres), but also by the facilitator, who drew attention to such actions. HELPERR changed from something to remember to something to envision performing parts of, depending on what others were doing in this particular situation. 
This involved varied epistemic levels of mediation, each of which was imbued, through the particular approach to debriefing, with relational qualities. The debriefs addressed questions of What? Who? When? Why? and Where to?, in addition to the 'How? In what order?' that HELPERR most explicitly denotes. New connections between 'I', 'you' and 'we' expanded participants' understandings of what happened, why actions and interactions were of consequence, and of what could or should happen in future.

Because shoulder dystocia is so unpredictable yet serious, the yearn for standardised practice is perhaps unsurprising. "Successful management of shoulder dystocia requires a rapid, standardized, and coordinated response" (McArdle et al. 2018, 191). Cornthwaite et al. (2015; quoted above) applaud the value of tools such as HELPERR in standardising practice, facilitating the performance of 'correct actions'. The term 'systematic', favoured by Baxley and Gobbo et al. (2012), has similar connotations.

However, some have questioned this. Reed (2015) argues that HELPERR is only relevant (at best) for shoulder dystocia cases encountered with a reclined mother on a bed; births can happen in confined spaces where mothers are upright. There is no standard patient, womb size, foetus, or size of the practitioner's hand when trying to release the shoulder. ${ }^{3}$

Writing more generally about healthcare practices, Reichenpfader et al. (2018) show that 'standardised' practice functions in everyday work through practitioners' collective reflexive work in which meaning is co-constructed in situationally specific ways, and where local adaptations are crucial. Our analysis shows that the quest for standardisation in response to shoulder dystocia is fruitful only up to a point: agile and responsive practices are needed alongside those of a systematic and predictable nature. 
This has important implications for understanding the function of protocol-based tools such as HELPERR, and how to approach continuing professional education.

HELPERR provides necessary commonality and stability, but PROBE promotes collective dynamics that mean the response to shoulder dystocia may be different every time. PROBE's adaptations of Steinwachs' (1992) three-stage approach to debriefing (Table 3) are central to this accomplishment, expanding the epistemic level of mediation by HELPERR to drive co-authorship of practice forward. Safe delivery requires more than practitioners having 'therapeutic algorithms in mind' (Monod et al. 2014, 4). The conceptualisation of HELPERR in terms of collective double stimulation addresses a missing 'we' in understandings of how to frame continuing professional education around shoulder dystocia.

In dialectic fashion, HELPERR both relies on and enables connections between practitioners as it is used in-between them. Double stimulation through PROBE unleashes this 'connective potential' (Kuiper 2018) of what would otherwise be a highly individualised practice tool. Debriefs highlighted changes in responsibilities and expanded each person's role, particularly around the need to verbalise what was being done. The shift from tool use privately by individuals to tool use between members of a team relies on such externalisations. The account of what happened (descriptive round), exploration of why actions mattered (analytic round), and projection into the future (application round) all highlighted dynamic webs of actions, re-actions and interactions.

Implicit in much of the prior work done on preparing practitioners for shoulder dystocia is the assumption that HELPERR functions as a memory tool that practitioners access to guide appropriate, standardised actions. Program evaluations have tended to focus on individual knowledge, confidence, competence, and compliance with 
prescribed actions. Recognition that safe management of shoulder dystocia requires an explicit focus on how the birthing team work together (Cornthwaite et al. 2015) demands new understandings of how tools such as HELPERR work to inform approaches to continuing professional education. A Vygotskian conceptualisation of HELPERR in collective double stimulation addresses this.

This approach first frames shoulder dystocia as a problem involving a conflict of motives that must be resolved through auxiliary means, delivering the foetus quickly and without injury. Crucially, the analysis reveals that education focused simply on being able to remember HELPERR and correctly perform the actions it prescribes, will not address what is demanded of professionals in collectively responding to the dilemma of shoulder dystocia. PROBE takes HELPERR beyond functions as a memory and sequencing tool, securing it as a means to co-author agile, collective actions.

\section{Conclusions}

Effective continuing professional education is crucial to improving outcomes in shoulder dystocia. Tools such as HELPERR form an obvious focus for such endeavours, but pedagogies that focus on memory and individual performance are insufficient. Despite recent recognition of the relational demands that shoulder dystocia places on professionals (Cornthwaite et al. 2015), there are few studies that reveal the mechanics of educational approaches associated with sustained positive outcomes in clinical practice (an exception being Hopwood et al. in press). PROBE has precisely such empirical support (Dahlberg et al. 2018) and thus forms a valuable focus. Our approach to exploring the pedagogies of simulation and debrief in PROBE has revealed the relevance and significance of double stimulation as a principle of volition action through which people escape conflicted situations (see Sannino 2015b; Vygotsky 1997). 
Approaches to training that focus on practitioner recall, confidence, and occurrence of actions as prescribed by HELPERR uphold a logic of mediation at an individual level (e.g. Draycott et al. 2008; Monod et al. 2014). The protocol mediates conflicts encountered and resolved by individuals in parallel as they externalise the mnemonic through actions performed in correct sequence.

Our analysis goes further by showing how PROBE transforms HELPERR into a tool that mediations actions in-between the team. The solution to the conflict ceases to be an answer to the question 'How do I act?' driven by an auxiliary motive to follow the protocol. Instead it rather answers 'How do we interact?' through a motive to relationally enact the protocol. PROBE activates double stimulation as principle through which collective volitional action emerges. But what of transformative agency?

Virkkunen refers to agency as breaking away from the given frame of action and taking the initiative to transform it' $(2006,49)$. Transformative agency arises when a focus on isolated problems is replaced with a focus on collective responses (Virkkunen 2006; Engeström et al. 2014). We suggest there are several indications that PROBE is indeed promoting transformative agency through double stimulation (TADS) among healthcare professionals in their response to shoulder dystocia. The 'given frame' would be HELPERR as a private memory tool, and tool that promotes standardised actions performed by individuals among others. PROBE helps practitioners break away from this frame, transforming it into a tool that promotes agile, collective action in which individuals attune to, anticipate, and connect with others.

Key to TADS is expansive transition from individual initiative to collective actions (Engeström et al. 2014). This is precisely what PROBE does, supporting participants to co-author their actions, collectively shaping and giving them direction. In the analysis we highlighted how participants envision new ways of working together, 
and commit to concrete, but not rigid actions. Such envisioning and commitment are two of six kinds of or expressions of transformative agency that have been noted in Change Laboratory studies (Engeström et al. 2014; Haapsaari \& Kerusuo 2015; Haapasaari et al. 2016). Haapasaari et al. note:

Actions and expressions of transformative agency emerge when people are placed in demanding situations and are given an opportunity to analyse, envision and redesign their activity collaboratively, with the help of mediating conceptual instruments. $(2016,259)$

This succinctly and accurately conveys how PROBE works. The simulation places practitioners in the extremely demanding situation of shoulder dystocia, and the debriefs provide them with opportunities to redescribe practice through collective narratives, analyse it, jointly envision future actions, and commit to future actions with others.

Haapasaari and Kerusuo (2015) found the connection between volition and conflicts of motives, understood in Vygotskian terms as a process of controlling one's own behaviour (after Sannino 2015a, 2015b) was an important aspect of sustaining transformative agency in their Change Laboratory research. Our study has demonstrated similar connections in the context of continuing professional education: double stimulation can be harnessed pedagogically as a principle of not just of volitional action, resolving isolated instances of conflicting motives, but of transformative agency, wherein professionals co-author their actions and interactions, giving new collective shape and direction to their practices.

This expands our understanding of TADS and provides a conceptual road-map to inform training offered to practitioners in a range of settings where teams are required to resolve conflicts of motives through use of protocols. Ongoing education for 
professionals responding to complex healthcare emergencies can be a means to foster transformative agency through double stimulation. The key lies in expansive tool use, across diverse epistemic levels, to address the relational demands of agile, responsive practice.

\section{Endnote}

${ }^{1}$ An adapted version of the ALSO HELPERR has been implemented in some settings, in which the first E refers to 'End pushing' (mother to stop pushing), the first R refers to 'roll over', and the second R to 'refer' to an obstetric unit if the prior moves are unsuccessful (Jenkins 2014; Huntley \& Dickson Smith 2017). The most recent RCOG (2012) guidelines use an algorithm that directly mirrors HELPERR but do not mention the mnemonic itself. The original version is the one used in the research setting, and which remains part of the ALSO course materials as of 2017; HELPERR in this paper refers to the ALSO HELPERR.

${ }^{2}$ Due to the number of nursing assistants on staff, not all simulation teams have a nursing assistant, in which case aspects of the role are substituted by the facilitator.

${ }^{3}$ HELPERR takes as a starting point a mother lying on her back on a bed; this is a common but not universal birthing practice, as there are many cultural, institutional and individual variations in the embodiments and materialities of birth. 


\section{References}

Baxley, E. G. \& R. W. Gobbo. 2004. "Shoulder dystocia.” American Family Physician 69 (7): 1707-1714.

Chauhan, S. P., R. Gherman, N. W. Hendrix, J. M. Bingham \& E. Hayes. 2010. "Shoulder dystocia: comparison of the ACOG practice bulletin with another national guideline." American Journal of Perinatology 27 (2): 129-36. doi: http://dx.doi.org/10.1055/s-0029-1224864

Cornthwaite, K., J. F. Crofts, T., Draycott, D. Siassakos \& C. Winter. 2015. Training for Obstetric Emergencies: Prompt and Shoulder Dystocia. London: The Health Foundation.

Crofts, J. F., C. Bartlett, D. Ellis, L. Hunt, R. Fox \& T. J. Draycott. 2006. “Training for shoulder dystocia: A trial of simulation using low-fidelity and high-fidelity mannequins." Obstetrics \& Gynecology 108 (6): 1477-1485. doi: 10.1097/01.AOG.0000246801.45977.c8

Crofts, J. F., D. Ellis, T. J. Draycott, C. Winter, L. P. Hunt \& V. A. Akande. 2007. "Change in knowledge of midwives and obstetricians following obstetric emergency training: A randomised controlled trial of local hospital, simulation centre and teamwork training." BJOG: An International Journal of Obstetrics \& Gynaecology 114 (12): 1534-41. doi: http://dx.doi.org/10.1111/j.14710528.2007.01493.x

Crofts, J. F., R. Fox, D. Ellis, C. Winter, K. Hinshaw \& T. J Draycott. 2008. "Observations from 450 shoulder dystocia simulations." Obstetrics \& Gynecology 112 (4): 906-912. doi: 10.1097/AOG.0b013e3181865f55

Crofts, J. F., C. Winter \& M. C. Sowter. 2011. "Practical simulation training for maternity care - where we are and where next." BJOG: An International Journal of Obstetrics \& Gynaecology 118 Suppl 3: 11-6. doi: http://dx.doi.org/10.1111/j.1471-0528.2011.03175.x

Draycott, T. J., J. F Crofts, J. P. Ash, L. V. Wilson, E. Yard, T. Sibanda \& A. Whitelaw. 2008. "Improving neonatal outcome through practical shoulder dystocia training." Obstetrics \& Gynecology 112 (1): 14-20. doi:

10.1097/AOG.0b013e31817bbc61 
Engeström, Y. 2007. "Enriching the Theory of Expansive Learning: Lessons from Journeys Towards Coconfiguration.” Mind, Culture and Activity 14 (1-2): 23-29. doi: http://dx.doi.org/10.1080/10749030701307689

Engeström, Y. \& A. Sannino. 2013. “La volition et l'agentivité transformatrice: perspective théorique de l'activité." Revue Internationale du CRIRES: Innover dans la Tradition de Vygotsky 1 (1): 4-9.

Engeström, Y., A. Sannino \& J. Virkkunen. 2014. “On the methodological demands of formative interventions." Mind, Culture, and Activity 21 (2): 118-128. http://dx.doi.org/10.1080/10749039.2014.891868

Fahey, J. O. \& H. E. Mighty. 2008. "Shoulder dystocia: Using simulation to train providers and teams.” Journal of Perinatal \& Neonatal Nursing 22 (2): 114-122. doi: 10.1097/01.JPN.0000319097.05415.1d.

Gobbo, R. W. \& E. G. Baxley. 2000. "Shoulder dystocia.” In Advanced Life Support in Obstetrics (ALSO) Provider Course Syllabus, edited by E. G. Baxley, M. Deutchman, L. Atwood, N. Murhy \& J. Yu. Leawood, Kansas: American Academy of Family Physicians.

Gobbo, R. W., J. Warren \& K. Hinshaw. 2017. "Shoulder dystocia.” In Advanced Life Support in Obstetrics (ALSO) Provider Manual, edited by L. Leeman, J. D. Quinlan, L. T. Dresang \& D. S. Gregory. Leawood, Kansas: American Academy of Family Physicians.

Goffman, D., H. Heo, S. Pardanni, I. R. Merkatz \& P. S. Bernstein. 2008. “Improving shoulder dystocia management among resident and attending physicians using simulations." American Journal of Obstetrics and Gynecology 294: e1-e5. http://dx.doi.org/10.1016/j.ajog.2008.05.023

Haapasaari, A., Y, Engeström, Y \& H. Kerosuo. 2016. “The emergence of learners' transformative agency in a change laboratory intervention." Journal of Education and Work 29 (2): 232-262. doi: http://dx.doi.org/10.1080/13639080.2014.900168

Haapasaari, A. \& H. Kerosuo. 2015. "Transformative agency: The challenges of sustainability in a long chain of double stimulation." Learning, Culture and Social Interaction 4: 37-47. doi: http://dx.doi.org/10.1016/j.lcsi.2014.07.006

Hope, P., S. Breslin, L. Lamont, A. Lucas, D. Martin, I. Moore, J. Pearson, D. Saunders \& R. Settatree. 2005. "Fatal shoulder dystocia: A review of 56 cases reported to the confidential enquiry into stillbirths and deaths in infancy." British Journal of 
Obstetrics and Gynaecology 105 (12): 1256-1261. doi:

http://dx.doi.org/10.1111/j.1471-0528.1998.tb10003.x

Hopwood, N., M. Abrandt Dahlgren \& K. Siwe. 2014. "Developing Professional Responsibility in Medicine: A Sociomaterial Curriculum." Chapter 12 in Reconceptualising Professional Learning: Sociomaterial Knowledges,

Practices, and Responsibilities, edited by T. Fenwick \& M. Nerland. London: Routledge.

Hopwood, N., M. Blomberg, G. Dahlberg \& M. Abrandt Dahlgren. In press. “Three principles informing simulation-based continuing education to promote effective interprofessional practice: Reorganizing, reframing and recontextualizing." The Journal of Continuing Education in the Health Professions.

Hopwood, N. \& B. Gottschalk. 2017. "Double stimulation 'in the wild': Services for families with children at risk." Learning, Culture and Social Interaction 13: 2337. doi: http://dx.doi.org/10.1016/j.lcsi.2017.01.003

Huntley, M. \& J. Dickson Smith. 2017. "Management of shoulder dystocia using the HELPERR mnemonic.” British Journal of Midwifery 25 (4): 240-244. doi: https://doi.org/10.12968/bjom.2017.25.4.240

Jenkins, L. 2014. "Managing shoulder dystocia: Understanding and applying RCOG guidance." British Journal of Midwifery 22 (5): 318-324. doi: https://doi.org/10.12968/bjom.2014.22.5.318

Kuiper, M. 2018. "Connective routines: How medical professionals work with safety checklists.” Professions \& Professionalism 8 (1): e2251. doi: http://dx.doi.org/10.7577/pp.2251

Mäkitalo, Å. 2014. “Categorisation.” In Analysing Social Work Communication: Discourse in Practice, edited by C. Hall, K. Juhila, M. Matarese \& C. van Nijnatten. London: Routledge.

McArdle, J., A. Sorensen, C. I. Fowler, S. Sommerness, K. Burson \& L. Kahwati. 2018. "Strategies to improve management of shoulder dystocia under the AHRQ safety program for perinatal care." Journal of Obstetric, Gynecologic, \& Neonatal Nursing 47: 191-201. http://dx.doi.org/10.1016/j.jogn.2017.11.014

Monod, C., C. A. Voekt, M. Gisin, S. Gisin. \& I. M. Hoesli. 2014. “Optimization of competency in obstetrical emergencies: A role for simulation training." Archives of Gynecology and Obstetrics 298 (4): 733-738. http://dx.doi.org/10.1007/s00404-013-3111-6 
RCOG. 2012. Shoulder Dystocia: Green-Top Guideline No. 42. London: Royal College of Obstetricians \& Gynaecologists.

Reed, R. (2015). Shoulder dystocia: the real story. Retrieved 30 May 2020 from https://midwifethinking.com/2015/05/13/shoulder-dystocia-the-real-story/

Reichenpfader, U., A. Wickström, P. Nilsen, M. Abrandt Dahlgren \& S. Carlfjord. 2018. "Medi(C)Ation work in the emergency department: Making standardized practice work." Professions \& Professionalism 8 (2): e2298. doi: http://dx.doi.org/10.7577/pp.2298

Sannino, A. 2015a. "The emergence of transformative agency and double stimulation: Activity-based studies in the Vygotskian tradition." Learning, Culture and Social Interaction 4: 1-3. http://dx.doi.org/10.1016/j.lcsi.2014.07.001

Sannino, A. 2015b. "The principle of double stimulation: A path to volitional action." Learning, Culture and Social Interaction 6: 1-15. http://dx.doi.org/10.1016/j.lcsi.2015.01.001

Sannino, A. 2016. "Double Stimulation in the waiting experiment with collectives: Testing a Vygotskian model of the emergence of volitional action." Integrative Psychological and Behavioural Science 50 (1): 142-173. doi: http://dx.doi.org/10.1007/s12124-015-9324-4

Sannino, A. \& A. Laitinen. 2015. "Double stimulation in the waiting experiment: Testing a Vygotskian model of the emergence of volitional action." Learning, Culture and Social Interaction 4: 4-18. doi: http://dx.doi.org/10.1016/j.lcsi.2014.07.002

Siassakos, D., J. F. Crofts, C. Winter \& T. J. Draycott. 2009. "Multiprofessional 'fireDrill' training in the labour ward." The Obstetrician \& Gynaecologist 11: 55-60. http://dx.doi.org/10.1576/toag.11.1.55.27469

Steinwachs, B. 1992. "How to facilitate a debriefing." Simulation \& Gaming 23 (2): 186-195. doi: http://dx.doi.org/10.1177/1046878192232006

Virkkunen, J. 2006. "Dilemmas in building shared transformative agency."@ctivités 3 (1): 43-66. doi: https://doi.org/10.4000/activites.1850

Vygotsky, L. S. 1997. The Collected Works (Vol. 4): The History of the Development of the Higher Mental Functions. New York: Plenum Press.

Weiner, C., L. Samuelson, L. Collins \& C. Satterwhite. 2014. "5-Year experience with PROMP (PRactical Obstetric Multidisciplinary Training) reveals sustained and progressive improvements in obstetric outcomes at a US hospital." American 
Journal of Obstetrics and Gynecology 210 (Supplement Issue 1): S40. doi: 10.1016/j.ajog.2013.10.094 


\section{Team 1}

Midwife 1: [To Mother] Push, push, everything you

can give, super good, you can do a little bit more

[The baby's head comes out]

Midwife 2: How's it going? Did it pull back or?

Obstetrician: Yes, it's pulling back, it's sucked back

Midwife 2: We turn off the drip [turns to switch off]

Obstetrician: Up with the legs [L]

Midwife 1: Push again

Obstetrician: No, we will make a cut [E; Assistant passes scissors to Obstetrician who cuts and passes back]. The drip is off, right?

[Midwife 1 has hands on the foetus, Obstetrician presses the mother]

Obstetrician: Now I will press the symphysis $[\mathrm{P}]$

Midwife 1: I'm putting my finger in [E], and I'm

trying to press, and here's the front shoulder... and

there's the rear shoulder, and I'm changing direction

[They switch roles so the Obstetrician now tries [E]

and Midwife 1 moves to apply pressure $[\mathrm{P}]$

Obstetrician: I' $m$ bringing the rear arm forward

Midwife 1: And now I'm pressing the symphysis

[Midwife 1 moves to press from the other side]

Obstetrician: That's alright, okay. [Baby delivered]

\section{Team 2}

The baby's head comes out]

Midwife 2: It's sucked back

Midwife 1: Yes, it's gone back. Shall we do

HELPERR?

Midwife 2: Switch off the drip, lower the mother's head [Midwife 1 does both of these]

Midwife 2: The legs are up [L], now you press [P]

[Midwife 1 applies pressure; Obstetrician looks at the clock; Midwife 2 beings the manoeuvres [E]

Midwife 2: It's not working, what do we do next? Midwife 1: Then we should release the shoulder Midwife 2: It's not working, you have to press Midwife 1: Shall we try the other direction? [Moves to the other side of the body]

Midwife 2: I can't do it. I'm trying the other direction. I've got my hand on the back shoulde Facilitator: It's one minute [since head birthed] Midwife 2: Ah thank you. [continues manoeuvres]. Now I've got it! [R1-shoulder is removed]... Now it's out! [Baby delivered]

\section{Team 3}

Midwife 1: No it's stuck, it feels like it is retracting

Obstetrician: Is it shoulder dystocia?

Midwes 1 and 2: Yes

Midwife 2: Then we turn off the drip

Midwife 1: I turn off the drip [does so]

??? Take the legs

Midwife 2: Is it $\mathrm{H}$ now?

Midwife 1: No we are at L

Obstetrician: And it is pressure [P] I will press here

Midwife 1: Pulling the head isn't working so I go in and try to release the shoulder [E]. Come on, push

Obstetrician: How is it working? Let me know when it's time to rotate

Midwife 1: No, it's loosening... turn it... see if I can get the arm. No. Will you try? [to Midwife 2]

Midwife 2: Will you try? [to Obstetrician]

Obstetrician: Should we roll over, or I will try once

Midwife 2: Try to get the arm [R1]

Obstetrician: I will try

Facilitator: It's been one minute

Obstetrician: We have to turn her over. It is the next step and we have to do it [R2]

Facilitator: You turn her and still don't get the baby out [the mannequin can't be rolled onto all fours]

Midwife 2: Let's try again... good it's coming. I have loosened the shoulder. [Baby delivered] 